\title{
Development and Design Principles of the Optimal Computer- and Robotic-aided Healthcare Systems for the Diagnosis, Treatment, and Rehabilitation
}

\author{
Vitali Guitberg \\ Alliance of Technology and Science Specialists \\ Toronto, Ontario, Canada \\ vguitberg@mail.com
}

\author{
Guennadi A. Kouzaev \\ Department of Electronic Systems \\ Norwegian University of Science and Technology-NTNU \\ Trondheim, Norway \\ guennadi.kouzaev@ntnu.no
}

\begin{abstract}
In this abstract, the principles of the development of the optimal computer- and robotic healthcare systems are proposed and considered as a means to improve the health service of the aging population. The results of the implementation of a new created diagnosis system Anamnesis are analyzed and found prospective to use the modified processors for handling the predicated flow of information. It is proposed to develop a robotic system for healthcare including the cyber-physical systems for the rapid manufacturing of medicine on-demand using microwaveassisted reactors.
\end{abstract}

Keywords-computer-aided diagnosis, computer-aided treatment, medical robots

\section{INTRODUCTION}

By this short description of our research and our future plans, it is supposed to attract the attention of the Federal and Provincial Governments of Canada and other countries, as well as the parliamentarians and general public for the acute problems in healthcare and to the means of radical improvements using the novel software and hardware diagnosis and treatment tools.

As known, the increase in retirement age leads to the growing of chronically unhealthy people at workplaces. Meanwhile, the hospitals and retirement houses encounter a severe shortage of qualified personnel. They do not always have enough funds available to improve this threatening humanitarian situation.

This contribution aimed to announce on our research on a novel system of integrated software and digitalized hardware performed at the Alliance of Technology and Science Specialists (ATSS, Toronto, Canada) together with the scientists and engineers from Canada, Norway, and Israel. This system will lead to the essential improvements of medical diagnosis, treatment, and rehabilitation of sick and older people.

First, in this conference abstract, the current state of the problem is analyzed on the example of the Canadian healthcare system, although, in general, the quality of medical service in Canada is one of the highest in the World [1]. Then, some new means are proposed for solution leading to the essential improvements of healthcare practice and economic outcomes in Canada and other countries having similar problems.

We consider our Optimal Computer-and Robotic-aided Healthcare Systems for the Diagnosis, Treatment, and Rehabilitation developed for implementation in the various applications. However, taking into account that the contemporary developed societies are aging, and, soon, the number of retired people will overcome the workable population, we put our attention to older people at first. This problem always exists and attracts attention to scientists, especially in developed countries [2].

In Russia, scientists and designers have been performed wide researches in the field of the climatology of the construction of the dwellings and public buildings. The goals of researches were to define norms and rules for creating a comfortable microclimate in the rooms with various assignments, including at medical buildings [3].

\section{HEALTHCARE REVIEW ON EXAMPLE THE CANADIAN SYSTEM}

\section{A. System Analysis of Medical Industry}

The Canadian Medical Industry has not been established as a united system of health-care services, and it has no optimal structure of it. Theoretical analysis and scientific approach can lead to rebuilding sub-systems of the Healthcare system, as a whole, and its units. The development of this optimal system can lead to an essential decrease in government expenses on healthcare.

Let's make a place for a direct citation on this problem: “... But our health care system is not set up to provide the best care for people with multiple chronic conditions. We need to move from fragmented patient care to an integrated approach that provides comprehensive, holistic care to people with multiple conditions. We need to put patients and families at the center of our health care system and ensure that all decisions are made with this in mind. ..."

https://www.carp.ca/2013/02/22/building-a-healthierontario-by-christine-elliott-opc-health-criticl C. Elliott, Ontario Progressive Conservative Deputy Leader Critic, Health \& Long Term Care, Sept. 2012).

\section{B. Medical Sciences}

The research in the field of medicine has fragmented again according to our best knowledge, due to the lack of unified systemic planning. It seems it is not due to poor planning but because medicine is a very complicated matter to be planned. New computer-aided means should be applied not only to planning but controlling the results of research activities. Due to that, there are so many gaps in medical science left undiscovered and unstudied. Besides, the insufficient studies of human health problems 
lead to multiple errors in diagnosis and treatment that lead to multiple deaths and economic loss [5],[6].

\section{Medical Education System and Quality of} Medical Specialists

The institutions of professional medical educations methodologically are not joined into one subsystem of preparation of the necessary specialists. As known, this leads to their periodical shortages and disproportions that influence the processes of the patient's diagnosis and treatment [5].

\section{Healthcare System Problems}

The Canadian Healthcare, as one of the main subsystems of the Medical Industry, currently also is not organized on a base of a holistic System Theory, but by political decisions based on economic situations. By the way, the medicine still is guided by provisions of the Canada Health Act, 1984. The country-wide announced task to improve healthcare service with the creation of the one single system of the EMR/HER (electronic medical records/health electronic records). The development of the system was run already more than 20 years ago, but it still has not been completed. Until today, there were established many independent medical institutions that, as it was planned, had to be interconnected by the EMR/HER. However, this has not been done, as it was planned. These institutions are:

1. The offices of the Family Physicians (FP) and other specialists

2. Walk-in-clinics

3. Laboratories

4. Pharmacies

5. Hospitals

6. Home care agencies

\section{E. Quality of Healthcare Specialists and Human Factors in Accurate Diagnosis and Treatment}

Although the purpose of service is the preservation of the health of the patients, this is not always achieved [7], and the reasons for this are the following:

- The medical specialists (MS) are not always able to accumulate and keep in mind all results of science although using modern information systems and databases - Medical specialists have too narrow medical education

- MS experience too high stress on the crowdy working place, and they not providing the necessary attention to the patient's complaints and taking them into account.

Due to these and many other reasons, MS are not able to make accurate enough diagnosis and adequate treatment of patients without a systematic transformation of decision-making techniques [8].

The Healthcare system is designed more beneficial for medical service providers than for patients. This "design" is reflected in the following:

- Due to the limitation of the number of patients for the Family Practitioner (FP) it is preferable to have patients with the chronical illness that require continual treatment

- The practice of requests from FP to provide an obligatory assessment of the patent's all body systems before making a diagnosis does not exist
- FP cannot control the patient's treatment at home by visiting him or communicate by phone, Skype, etc.; there is no time for direct contact between the doctor and patients [7].

- Pharmacists are urged selling as many as possible medication, that is not safe for patients [9],[10].

\section{F. Lab Examination Errors}

The main problem of the lab examinations is some unreliability of their results. They depend on not only the quality of the medical diagnostic equipment and used software but mostly from the professional level of the technicians, who work with them. Our experience shows that the lab examinations of the same body parts done by different technicians may show different results. The differences in results could be using different medical instrumentation.

Preparation of the report of the MS to the FP could take a long time, and its accuracy also depends on the MS professional level. So, there is no trustable control of the accuracy of the patient's health data, and this also is a source of heavy or even lethal mistakes [9].

\section{G. Pharmaceutical Problems}

First, the pharmaceutical industry produces drugs for treatment almost all illnesses with many harmful and dangerous side effects. Due to this, people consider the current Medical Industry as the "aggressive medicine" because, with such medications, some illnesses could be treated, but other illnesses could appear.

Second, the production of a huge number of pharmaceutical products for the treatment of the same symptoms (illnesses) makes for doctors too difficult and sometimes almost impossible to select the best (optimal) set of medications. It is one of the main reasons for making the doctor's errors.

Third, the number of medical drugs and devices that are selling by pharmacy depends on the number of medical/doctors' errors: more errors - a larger number of goods would be sold [5].

\section{Proposed METHOdOlOGY IN DiAGNOSIS AND TREATMENTS}

In this section, we consider a methodology of the systematic approach in diagnosis and optimal treatment design developed by our group of specialists. The first work in this direction is published in late 80-th [3],[9]. This systematic approach can be considered as an alternative one to the current organization of healthcare in Canada. It consists of unlink institutes of independent medical professionals that aimed to treat the specified illness instead of the human organism overall.

Today, the FPs cannot take into account the patient's health conditions on the whole. They arranged their patients to medical specialists trained to treat specific problems, even they able to decide them by themselves. The picture of such a situation is seen at any hospital's emergency room. It is caused that an MD is not able to keep in its mind all diagnosis and treatment specifics of multiple complicated health problems of humans.

In this contribution, we are considering our project and preliminary results on the creation of a Computerized 
Recovery Adviser (ComRAD). It is aimed to improve to some extent, the mentioned Healthcare System in Canada (See Figs. 1 and 2). It is an analytical system assigned for performing the most accurate diagnosis and the optimal treatment using Human-Computer interaction and IT-based software tools. Due to this, ComRAD will improve the diagnosis and treatment flow to make any health system essentially effective. It is supposed that ComRAD will be a part or subsystem thought of as "Intelligent Information and Expert/Design System” (II\&ES) ${ }^{\odot}$.

The mentioned II\&ES has been designed on a base of a unique "Methodology of Computerized Systems Analysis and Design" (MCAD) as the common Intelligent and Information System for the use in any subject domains to optimize the process of designing any object and for obtaining the optimal result.

It means that the II\&ES is assigned for use in any field of human activities to obtain the best possible result. The globality of the project of the II\&ES (Fig. 1) is in its unique components: "Electronic Knowledge Base" $(\mathrm{eKB})^{\odot}$, "Operating Database" (ODB), and "Complex of Methodical Software" $(\mathrm{CoSMoS})^{\odot}[3]$.

The eKB was created using the computerized method of the System Analysis for the population of the scientific information into its ontology; i.e., the ODB is an advanced substitute of the EHR/EMR. For the management of the eKB and ODB, as permanently extending components of the II\&ES, the CoSMoS software complex was developed.

Dr. V. Guitberg published the first works in this subject in the late of the last century's 80s. Being a universal tool, they were implemented in some projects in the field of building construction until the early 1990s.

In Canada, starting since 1992, further developments of the II\&ES were performed together with I. Elkhinovich, a system engineer and computer programmer. For this goal, a company named "Designing of Intellectual Systems Knowledge" (DISK Inc.) was established and registered.

DISK Inc developed the ComRAD tool in cooperation with a group of specialists (medical doctors, computer science specialists, and others) and members of the nonfor-profit organization "Alliance of Technology and Science Specialists of Toronto" (ATSS Inc). E. Kozhemyakin has developed the unique CoSMoS software complex.
The initial part of the ComRAD is a pre-diagnostic test "Anamnesis"(C) that designated for an assessment of health conditions of all body systems of the patient to prevent (See Figs. 1 and 2). By using "Anamnesis," it will also become possible to avoid or, at least, reduce the MD's errors and also make unnecessary and unpleasant lab examinations.

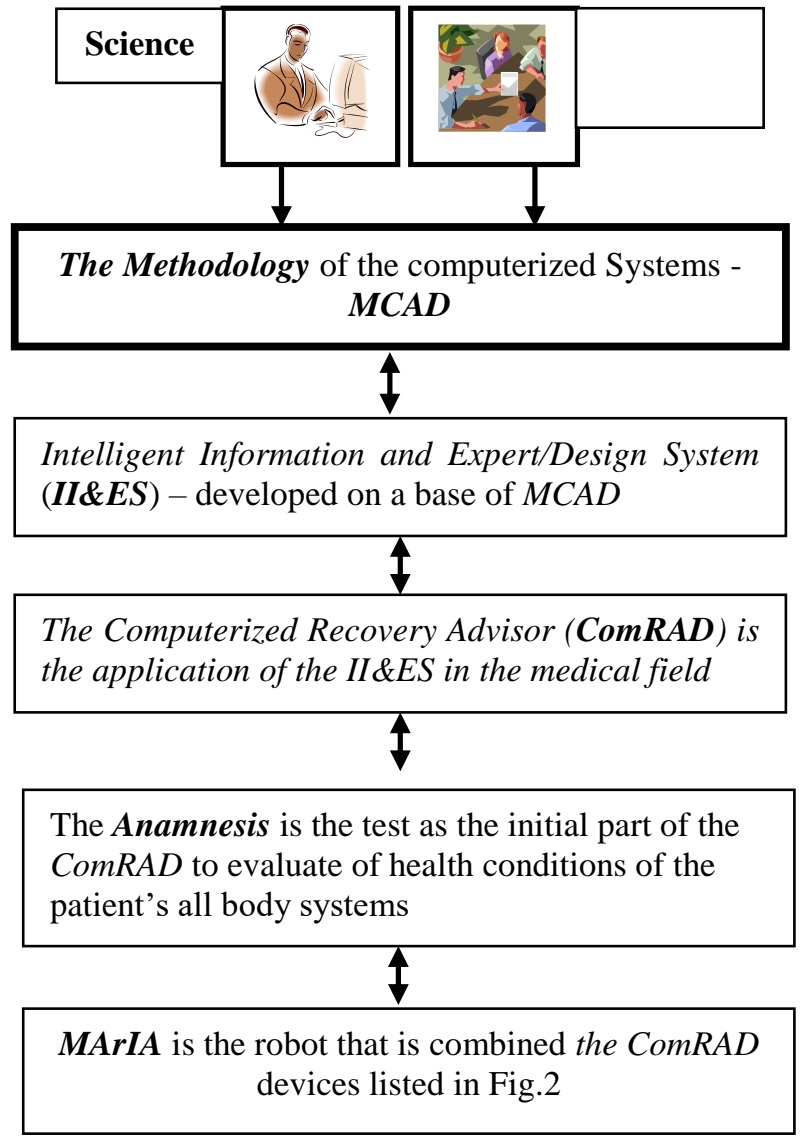

Fig. 1. The scientific base and practical tools (MoscowRussia, Toronto-Canada, Trondheim-Norway, Tel-Aviv-Israel.)

According to our methodology (Fig. 1), the ComRAD/ Anamnesis designed for using in several options: for MD's serious illnesses offices, at hospitals, patient home care, etc. It allows the prevention of diseases by using the ComRAD/Anamnesis, a priority task of MDs. It would allow to FPs performing remote home care of their patients by monitoring and control of the patient's health condition.

Fig. 2. The Optimal Computer-aided process for Health Diagnosis, Treatment, and Rehabilitation (ComRAD).

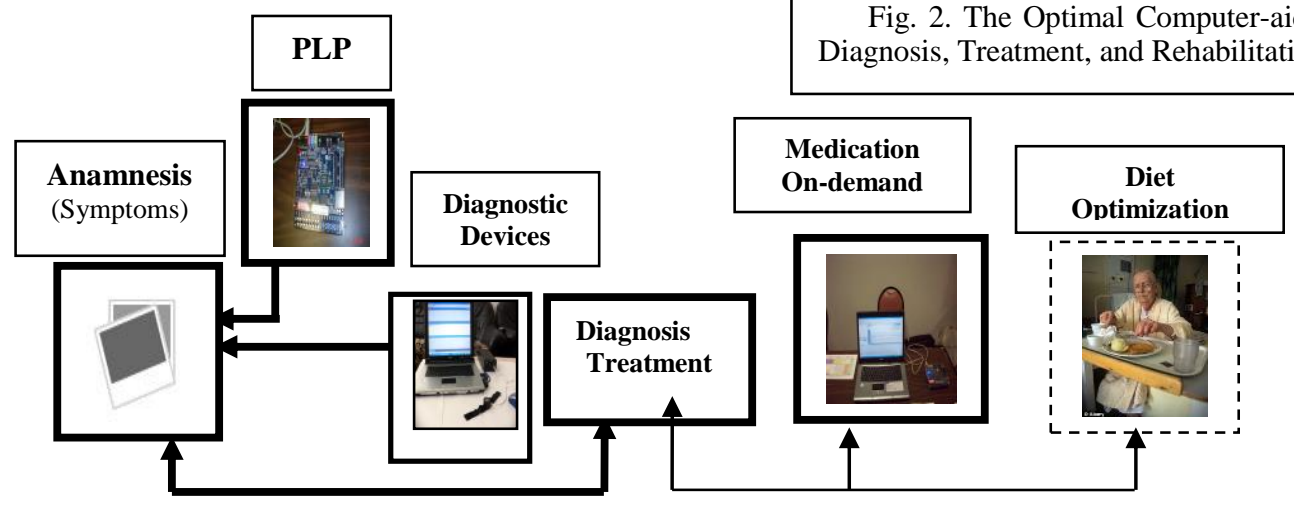


The functioning of all devices, associated with the ComRAD's components, placed on the MrIA would be provided by the eKB, CoSMoS, and ODB with connecting them to their central counterparts.

The proposed pre-diagnostic test Anamnesis that includes 153 symptoms and allows within 5-10 minutes to analyze the state of health of all eleven human body systems (See Fig.1). Using the test's results, the CoSMoS would get from the eKB the necessary information (instruction) automatically. It should be used by the family physician (FP) for the treatment of a patient. It becomes possible due to using the method of systems analysis during the population of the scientific data at the eKB and in using them in the process of treatment. In designing the Anamnesis, it has been used the Questionnaire approved by the American Medical Association (AMA) and Chiropractors' Questionnaire.

Due to combining the MrIA with several devices, it will be possible to not only provide testing with the "Anamnesis," but also perform several physical exams in the process of monitoring of treatment, at-home cares, in a hospital's ER, and waiting rooms of doctor's office [10] .

\section{DETAILED DESCRIPTION OF THE PROPOSED SYSTEM}

We consider that all doctors (doesn't matter in a hospital or their offices) are carrying the highest responsibility for the life of their patients. Then, we state in our project the principle that "doctor is first." It means that we aim to provide the MDs with the unique assisting computer technology ComRAD to make their work more effective and less stressed.

We also believe that no other way exists for the design of the "Optimal Healthcare System." The existing single diagnostic devices and the EMR/EHR programs do not possess advantages as the ComRAD [7].

On the future stage of the ComRAD project development, our team is planning to extend the MrIA's functions for the development of a complex of devices for the treatment and home care of the patients with chronic illness, especially for seniors and people with disabilities. This ComRAD/MrIA, should combine the followings physical and electronic components (Fig. 2):

- Medical electronic Knowledge Base (eKB) consists of the modular electronic ontology of the knowledge with universal architecture that allows to populate it with all kinds of scientific medical information.

- Operating Database $(\mathrm{ODB})^{\odot}-$ includes the patient's healthcare history and consists of modular electronic records that saved in the unique database with the architecture similar to the eKB (different from the EHR/EMR).

- $\operatorname{CoSMoS}^{\odot}$ - software complex that realizes the unique computerized method of System Analysis in the process of managing data population into the ontology of scientific knowledge of the eKB

- Diet optimization tool (under the development being in the research study).

- Wirelessly-connected sensors [12],[13] of different designs and physiological data acquisition (for instance, Initial Test We are considering that in the not seldom there is a severe shortage of MDs and nurses, the exploitation of robots it is supposed in our project. Together with S. Sutherland, who is the developer of the CrossWings Robot (CrossWings Inc, Toronto, Canada), we have developed a conceptual project of a more advanced robot. It is called "My Robotic Intelligence Assistant" (MrIA). It must combine all existing and still developing devices (See Fig. 2). Due to this, such robots to be adapted to any option of implementation: such as for diagnosis, monitoring of the health conditions and treatment, home care, and rehabilitation of the patients. "Anamnesis" and "Puls-MedicAnalytics" (Israel) as an analog of "Heart Watch" (China) - diagnostic devices), including the micro- and millimeter-and submillimeterwave radiovision systems for safe distant diagnosis of some diseases and physiological states of human bodies [14],[15].

- Instrumentation and software tools for the analysis of bioimages in different parts of the electromagnetic spectrum [2].

- Predicate-logic-based processors (PLP) for micro-parallel predicated information flows from the biosensors and medical database banks. The already developed processors [15],[16],[17] are working now on binary sentences instead of single bits that allow implementing the operations of artificial intelligence on binary hardware level with the acceleration of data processing.

- Robotic desktop hardware for "Medications on Demand." This tendency in the pharmaceutical industry is required by hospitals and small treatment and rehabilitation centers to decrease the amount of non-consumed drugs and to manufacture only the tailored amount of prescribed medicine. It supposed that this chemical hardware would be computercontrolled and flexible using the Internet-ofThings means in a human-less environment [18]. This new application of pharmaceutical hardware requires fulfillment of very strict conditions on safety and flame- and leakage-less manufacturing. Additional demands are on the acceleration of chemical processes. Very often, it is realized using the application of microwaves for direct heating of reagents and polar or conducting solvents. It allows excluding the high-temperature electric heaters or /and highboiling-point toxic heating liquids with the noticeable acceleration of many reactions in microwave irradiation. For instance, in [19], a new reactor is proposed where the liquid is heated with microwaves in a thin sub-millimeter gap between the stator and dielectric rotor. It allows reaching homogeneous heating of liquids on microlevel that causes the predictable, rapid chemical yield which is especially important in tailored fine chemistry. 


\section{CONCLUSION}

In this conference abstract, the principles of the optimal healthcare system have been developed. In the framework of this research, a methodology of the development of the main components of the Computerized Recovery Adviser (ComRAD)(C has been proposed. It has been created the main part of the Intelligent Information and Expert/Design System" (II\&ES)(C - electronic Universal Knowledge Base (eUKB). It has been developed a demo-version of a diagnosis system Anamnesis verified with the illness diagnosis of disease for more than ninety individuals. The developed software was presented in several hospitals in Canada. Further developments are for enhancing the Anamnesis, development of the Operating DataBase (ODB) for the medical field, and the integrating of sensors with wireless links and computers for continuous diagnosis of ill individuals.

A serious attention is supposed to pay on the development of the hardware for medicine-on-demand for small treatment centers under the strict control of electronic prescriptions and pharmaceuticals consumption.

\section{ACKNOWLEDGMENT}

Authors thank more than ninety individuals who took part in testing the Anamnesis during the demo stage research in 2008 and the main stage of the implementation of this software tool (2008-2010).

We are thankful to our colleagues, O. Amurguev, S. Sutherland, and J. Sherrington, who actively participated in presentation of this System to the following organizations: VentureLab, Healthcare Ecosphere (Markham, GTA), Baycrest Hospital, Rotman Research Institut (North York, Toronto), and Southlake Regional Health Centre (Newmarket, ON).

\section{REFERENCES}

[1]. C. Simpson, D.M.C. Walker, D. Drummond, D. Sinclair, and R. Wilson, "How healthy is the Canadian health-care system?" The Conversation, Sept. 25, 2017. http://theconversation.com/how-healthy-is-the-canadianhealth-care-system-82674, seen on Nov. 15, 2019.

[2]. P.M. de Azevedo-Marques, A. Mencattini, M. Salmeri, and R.M. Rangayyan, Medical Image Analysis and Informatics: Computer-Aided Diagnosis and Therapy, CRC Press, 2017.

[3]. V. Guitberg, The Systematic Design (in construction), In Russian, Stroyizdat, Leningrad, USSR,1987.

[4]. M. Mesarovich, D. Macko, and Y. Takahara, The Theory of Multi-level Hierarchical Systems, Academic Press, 1970

[5]. T. Blackwell, "Inside Canada's secret world of medical error: There is a lot of lying, there's a lot of cover-up," National Post, Jan. 15, 2015.

[6]. Z. Damania, "We need to demand technology that lets doctors be doctors," Tech, Feb. 1, 2016, seen at https://www.kevinmd.com/blog/2016/02/need-demandtechnology-lets-doctors-doctors.html on Dec. 07, 2019.

[7]. V. Guitberg, No Alternative, Albion, UK, 2012.

[8]. "What is Human Factors in Healthcare?" https://www.medicalhumanfactors.net/about-us/what-ishuman-factors/, seen on Nov. 16, 2019.

[9]. J.L. Howe, K.T. Adams, A.Z. Hettinger, and R.M. Ratwani, "Electronic health record usability issues and potential contribution to patient harm," J. Amer. Med. Assoc., vol. 319, pp. 1276-1278, 2018

[10]. "Fraudulent prescriptions: a serious risk to the NHS." https://cfa.nhs.uk/about-nhscfa/latest-news/fraudulentprescriptions-serious-risk-to-nhs, seen on Nov.16, 2019.

[11]. E. McCann, "Deaths by medical mistakes hit records. The way IT is designed remains part of the problem," HealthCare IT News, July 18, 2014. Seen at: https://www.healthcareitnews.com/news/deaths-by-medicalmistakes-hit-records on Dec. 12, 2019.

[12]. L.M. Najeh, "Study and analysis of sensors in a smart hospital system," J. Sensors, vol.1, pp. 1001, 2019

[13]. G.V. Angelov, D.P. Nikolakov, I.N. Ruskova, E. E. Gieva, and M. L. Spasova, "Healthcare sensing and monitoring," In: Enhanced Living Environments, pp. 226-262, Springer, Berlin, 2019.

[14]. E.E. Godik and Yu.V. Gulayev, "Functional imaging of the human body: dynamic mapping of physical EM fields signals a breakthrough in medical diagnostics," IEEE Eng. Med. Biol. Mag., vol. 10, pp. 21-29, 1991.

[15]. G.A. Kouzaev, Application of Advanced Electromagnetics. Components and Systems, Springer, Berlin, 2013.

[16]. G.A. Kouzaev and A. Kostadinov, "Predicate gates, components and a processor for spatial logic," J. Circ., Syst. Comp., vol. 19, pp. 1517-1541, 2010.

[17]. A. Kostadinov, V. Guitberg, M. Olavsbraten, and G.A. Kouzaev, "MultiLogics Gates," Proc. IEEE Int. Seminar Electron. Dev. Design\&Production, pp. 1-3, Apr. 23-24, 2019, Prague, Czech Republic.

[18]. J. Li, S.G. Ballmer, E.P. Gillis, S. Fujii, M.J. Schmidt, A.M.E. Palazzolo, J.W. Lehmann, G.F. Morehouse, and M.D. Burke, "Synthesis of many different types of organic small molecules using one automated process," Science, vol. 347 pp. 1221-1226, 2015

[19]. G.A. Kouzaev, "The separate supply of microwave and mechanical energies to liquid reagents in coaxial rotating chemical reactors," Pat. Appl. GB1704095.7, Search. Pat. J., vol. $6748,2018$. 